\title{
A FIELD-CANCELLATION ALGORITHM FOR CONSTRUCTING ECONOMICAL PLANAR PERMANENT MAGNET (PM) MULTIPOLES WITH LARGE HIGH-QUALITY FIELD APERTURES ${ }^{*}$
}

\author{
R. Tatchyn \\ Stanford Synchrotron Radiation Laboratory, SLAC, Stanford, CA 94309, USA
}

Abstract

In recent years studies have been initiated on a new class of multipole field generators consisting of cuboid planar permanent magnet (PM) pieces arranged in biplanar arrays of 2 -fold rotational symmetry. These structures, first introduced for Free Electron Laser (FEL) applications, are based on reducing the rotational symmetry of conventional $\mathrm{N}$-pole field generators from $\mathrm{N}$-fold to 2-fold. One consequence of this reduction is a large higher-multipole content in a planar PM multipole's field at distances relatively close to the structure's axis, making it generally unsuitable for applications requiring a large high-quality field aperture. In this paper we outline an economical field-cancellation algorithm that can substantially decrease the harmonic content of a planar PM's field without breaking its biplanar geometry or 2fold rotational symmetry.

\section{INTRODUCTION}

In recent years, the development of a novel class of PM multipole (N-pole) field generators has been initiated at SSRL $[1,2,3,4]$. The basic construction principle, depicted in Fig. 1, is to arrange $\mathrm{N}$ rectangular PM pieces, magnetized with their easy axis perpendicular to two opposed faces, into a biplanar array with N/2 pieces per plane. In general, while the pieces in each quadrant of the $\mathrm{x}-\mathrm{y}$ plane can have arbitrary dimensions, x-placements, and magnetizations, the overall structural and field geometries possess symmetry with respect to the $y-z$ and $\mathrm{x}-\mathrm{z}$ planes, and the normal convention is to have each of the two sets of magnet surfaces closest to the $x-z$ plane be coplanar. If we postulate an ideal N-pole generator to be a structure with $\mathrm{N}$-fold rotational symmetry [5], the essential concept of planar PM multipoles is seen to be based on the reduction from $\mathrm{N}$-fold to 2-fold rotational symmetry.

The entire class of planar PM multipoles can be grouped into two families: 1) $\mathrm{N}=4 \mathrm{n}$; and 2) $\mathrm{N}=4 \mathrm{n}-2$; where $\mathrm{n}$ e $\{1,2, .$.$\} . Thus, the first family includes the$ quadrupole (quad), octupole, dodecapole, etc., and the second family includes the dipole, sextupole, decapole, etc. From Fig. 1 it is seen that the basic criterion for this classification is field symmetry, viz., the $\mathrm{N}=4 \mathrm{n}$ family has

\footnotetext{
* Work supported in part by the Department of Energy Offices of Basic Energy Sciences and High Energy and Nuclear Physics, and Department of Energy Contract DE-AC03-76SF00515.
}

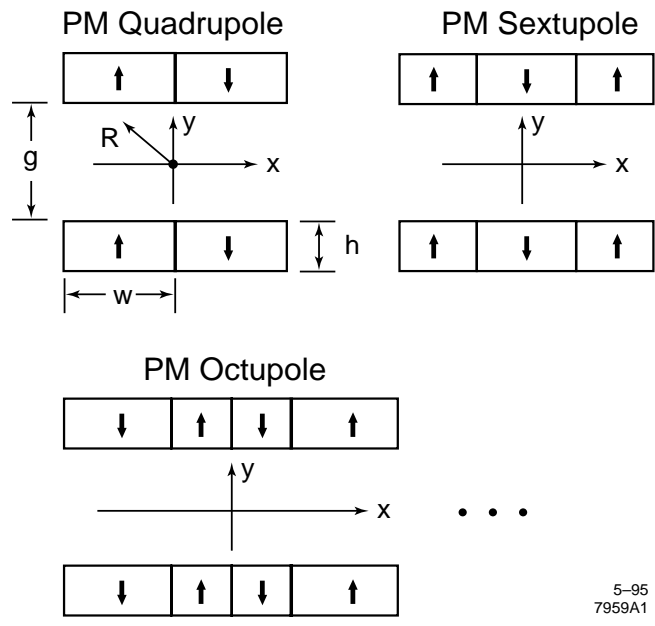

Fig. 1. Planar PM multipoles composed of pieces of equal height (h), with no lateral spacing between pieces. Symmetry axis ( $\mathrm{z}$ axis), along which all the PM pieces have equal length $\mathrm{L}$, is perpendicular to the page.

fields with odd symmetry vs. $\mathrm{x}$, while the $\mathrm{N}=4 \mathrm{n}-2$ family has even field symmetry. For each family, the corresponding magnetic scalar potential (for $\mathrm{L}>\mathrm{g}$ ) in the vicinity of the symmetry axis can be approximated by a real Taylor series expansion as follows:

$$
\begin{aligned}
\phi_{4 n} \cong & C_{11} x y+E_{13}\left(x y^{3}-x 3 y\right) \\
& +G_{15}\left(3 x y^{5}-10 x^{3} y^{3}+3 x^{5} y\right)+\ldots ;
\end{aligned}
$$

and

$$
\begin{aligned}
\phi_{4 n-2} \cong & B_{01} y+D_{21}\left(3 x^{2} y-y^{3}\right) \\
& +F_{41}\left(5 x^{4} y-10 x^{2} y^{3}+y^{5}\right)+\ldots ;
\end{aligned}
$$

where the subscripted capital coefficients are functions of the PM parameters. To configure a given $\mathrm{N}$-pole, the dimensions, spacings, and magnetizations of the $\mathrm{N}$ pieces must be designed to eliminate all the multipole coefficients lower than the desired leading $\mathrm{N}$-pole coefficient. As formulas (1) and (2) explicitly show, the field of each such N-pole will not only exhibit its leading $\mathrm{N}$-pole coefficient, but will also contain an infinitude of higher-pole terms with strengths proportional to increasing integral powers of distance $(\mathrm{R})$ from the $\mathrm{z}$ axis.

In the following sections, an economical algorithm for removing the dominant members of these higher terms as a means of restoring the high-quality field aperture of a planar PM multipole to values comparable to those of a conventional structure, is outlined. 


\section{APPROACHES TO PLANAR MULTIPOLE FIELD QUALITY IMPROVEMENT}

In contrast to a rotationally $\mathrm{N}$-fold symmetric $\mathrm{N}$-pole, whose pole surfaces can be shaped to approximate to the optimum equipotential contours [6], the freedom of adjusting the parameters of planar PM multipoles to attain a comparable degree of field quality is rather limited. Specifically, the shapes of the PM pieces (all rectangular cross sections) cannot be modified, forcing all equipotential surfaces to be planar. Secondly, confinement to a plane prevents the rotation of the pieces as a means of approximating to a curved 2-dimensional equipotential contour. Under these constraints, the principal means of improving the field quality has to be associated with the adjustable degrees of freedom of the planar PM multipole, namely, the number, dimensions, $\mathrm{x}$ placements, and magnetizations of the PM pieces. Approaching the problem from this perspective, eq's. (1) and (2) suggest a straightforward way of enhancing the field quality of a planar PM N-pole; namely, by the successive removal of its higher-pole field components [3].

The basic principle behind this approach is illustrated in Fig. 2. For an optimized planar PM quad with the field spectrum shown on the top left side, the octupole component

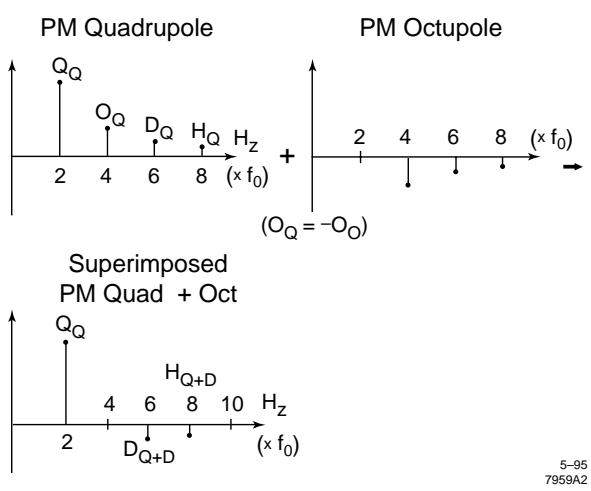

Fig. 2. Linear superposition of planar PM octupole and quadrupole rotating-coil spectra as a means of nulling the octupole component in the combined structure. $\mathrm{f}_{0}$ is the coil rotation frequency.

can be nulled by centering a planar PM octupole whose leading field component, $\mathrm{O}_{\mathrm{O}}$, is equal and opposite to the quad's octupole component, $\mathrm{O}_{\mathrm{Q}}$, over the symmetry axis of the quad. We note that since the potential is given by the same canonical form for each structure (viz., by eq. (1)), making $\mathrm{O}_{\mathrm{O}}=-\mathrm{O}_{\mathrm{Q}}$ will null the octupole component (E13 in our notation) for all values of R. Due to linear superposition, all the higher-pole components of the PM octupole will also tend to subtract from the corresponding higher-pole components of the quad. This procedure can be, of course, repeated to eliminate the next higher (dodecapole) component of the combined quad+octupole structure (see Fig. 3). Further repetition can be used to successively remove as many of the nexthigher (or indeed, arbitrarily-located) field components as desired. Consequently, the same method can be used to purify the field of any planar PM N-pole. It is easy to show that if an individual PM multipole is used for each cancellation, the total number of PM pieces required for a quad with $\mathrm{m}$ successively nulled next-higher components would be $4+6 m+2 m^{2}$, and the corresponding number of pieces for a sextupole would be $6+8 m+2 m^{2}$. Clearly, the straightforward application of the cancellation procedure, as described up to this point, requires: 1) the vertical height of the initial $\mathrm{N}$-pole structure to increase substantially; and 2) the number of pieces to go up rapidly with the required degree of purity. Both of these factors tend to li mit the practical implementability of this approach.
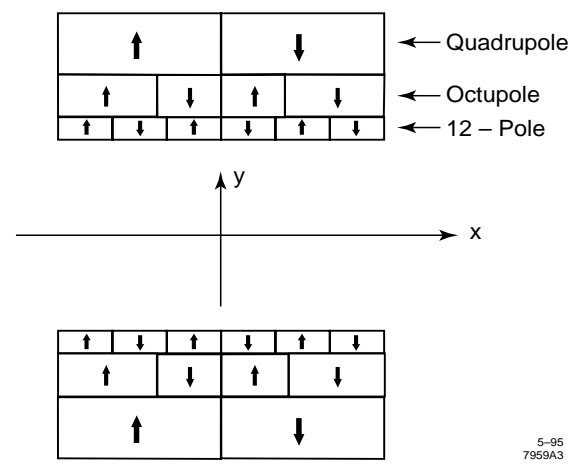

Fig 3. Planar PM quadrupole structure with nulled octupole and dodecapole field components.

\section{AN ECONOMICAL FIELD-CANCELLATION ALGORITHM}

The principle of the improved method (algorithm) to be introduced in the present paper can now be stated: by applying linear superposition to the PM magnet pieces themselves it is possible to generate fields equivalent to those of the improved structure of Fig. 3, but with a substantially reduced number of PM pieces, and without compromising the primary advantages of the basic N-pole structures of Fig. 1.

Referring to Fig. 3, the first requirement is that all the individual N-poles comprising the structure be of equal width. The second requirement is that all the PM pieces comprising the structure be of equal height. The third requirement, which underlies the superposition of the pieces is that each of the individual N-poles comprising the structure have the same gap. It is essential to note that under these constraints the field cancellations generated by the octupole and dodecapole can be maintained by suitable adjustments of the magnetization of their PM pieces. To illustrate the method, we can first consider the quadrupole and octupole structures in Fig. 3. Under the cited constraints, the octupole will be completely 
superimposed over the quadrupole, yielding a structure with only eight pieces. The magnetizations of these pieces, however, will now be the linear sum of the magnetizations of the superimposed quadrupole and octupole structures, yielding a structure with the original quadrupole field component and a completely nulled octupole component. This has now been accomplished with eight pieces instead of twelve.

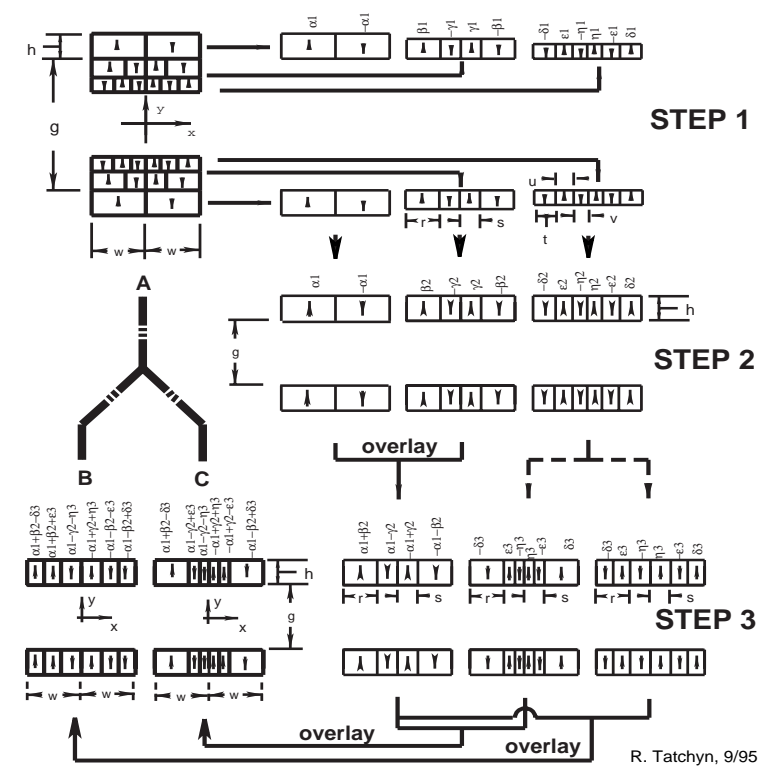

Fig. 4. Planar PM quadrupole field-cancellation algorithm for removing octupole and dodecapole components with a minimal number of pieces. Structures $\mathrm{B}$ and $\mathrm{C}$ are, apart from a scalar factor, equivalent to $\mathrm{A}$.

We next take this new structure and superimpose the dodecapole structure over it in the same fashion. The new constraint is that the total width of the two pieces to the right of the dodecapole's y axis must be equal to the width of the piece to the right of the y axis of the new eightpiece structure. With this condition, a new twelve-piece structure is generated which has the original quadrupole component and completely suppressed octupole and dodecapole components. This has now been accomplished with only twelve pieces instead of the 24 shown in Fig. 3. Continuing in this fashion, it is straightforward to show that a planar PM quadrupole with $\mathrm{m}$ successively nulled next-higher components requires $4+4 \mathrm{~m}$ pieces, and the corresponding number of pieces for the sextupole is $6+4 \mathrm{~m}$. In similar fashion, similar reductions in the complexity of higher $\mathrm{N}$-poles can be achieved. A schematic flow chart of the fieldcancellation algorithm described in the above paragraphs is shown in Fig. 4. In this picture the magnets are represented by equivalent magnetic charge sheets on their top and bottom surfaces, with the charge density magnitudes labeled by lower-case Greek letters. It is seen that a major penalty incurred by the proposed algorithm is the substantial reduction in the quadrupole strength of the reduced structures. As is evident for the quadrupole case, the algorithm generates two alternative paths; higher-Npole optimization will involve even greater numbers of possible implementation options. The different possible configurations evidently provide the opportunity for further optimization, such as, e.g., minimizing the maximum/minimum spread in the values of magnetization while maximizing the dominant (N-pole) component in the final reduced structure.

\section{SUMMARY}

An economical field-cancellation algorithm has been described which will allow the fabrication of bi-planar quadrupoles and sextupoles with high-quality fields using a manageably small number of PM pieces. For higher order $\mathrm{N}$-poles the number of pieces required to cancel a given number of successively-higher multipole components will also increase linearly; nevertheless, the practicability of fabricating octupoles and higher N-poles of this type should be considered a subject of continuing r\&d. Since the removal of a large number of successive multipole components essentially increases the transverse region over which the $\mathrm{N}$-pole's field is dominated by its leading $\mathrm{N}$-pole field component, the fabrication of quadrupoles and sextupoles of the type described in this paper should lead to their introduction in storage ring applications. One potentially important application in this area is as distributed focusing elements installed into very-short-period, small-gap undulators (e.g., as a FODO lattice) [7]. The installation is rendered feasible by the very small vertical height of the biplanar $\mathrm{N}$-poles (on the order of a millimeter), which, notwithstanding, doesn't prevent them from attaining focusing gradients on the order of several hundred $\mathrm{T} / \mathrm{m}$. If proven, this would allow short-period undulators of substantial length (viz., >>b) to be operated on storage rings, potentially transforming the optimality and economy of synchrotron radiation sources toward more favorable regimes.

\section{REFERENCES}

[1] R. Tatchyn, "Selected applications of planar permanent magnet multipoles in FEL insertion device design," Nucl.. Instrum. Meth. A341, 449(1994).

[2] R. Tatchyn, "Planar Permanent Magnet Multipoles for Particle Accelerator and Storage Ring Applications," IEEE Trans. Mag. 30(6), 5050(1994).

[3] T. Cremer, R. Tatchyn, "Planar Permanent Magnet Multipoles: Measurements and Configurations," in Proceedings of the 1995 Particle Accelerator Conference, IEEE Catalog No. 95CH35843, ms. FAP-20(on CD).

[4] J. Cobb and R. Tatchyn, "Tests of planar permanent magnet multipole focusing elements," Nucl.. Instrum. Meth. A347, 562(1994).

[5] D. C. Carey, "The Optics of Charged Particle Beams," Harwood, New York, 1987.

[6] K. Halbach, "Design of Permanent Multipole Magnets with Oriented Rare Earth Cobalt Materials," Nucl. Instrum. Meth. 169, 1(1980).

[7] R. Tatchyn, P. Csonka, A. Toor, "Perspectives on micropole undulators in synchrotron radiation technology," Rev. Sci. Instrum. 60(7), 1796(1989). 